\title{
Note on the Solution of Partial Differential Equations \\ by the Method of Reciprocation.
}

\section{By John M'Cowan, D.Se.}

In a paper " On the Theory of Long Waves " recently published," I drew attention to a point of some interest in the theory of the solution of partial differential equations by what is usually termed the method of reciprocation. As the subject was apart from the main object of the paper, however, I there noticed the peculiarity but briefly, and so I have thought that it might be of some use to enter into the matter here somewhat more fully. On first adverting to the point, it was my opinion that it could hardly have escaped the notice of those who had made much application of the method of solution by reciprocation, though I had not myself seen any reference made to it; but as I have not had my attention drawn to any such notice since the publication of the paper last March, it seems probable that it has not previously been discussed.

The method of reciprocation, due, I believe, to Legendre, is so well known that the following brief description may here suffice. By changing the variables in the given equation from $x, y$, and $z-$ employing the usual notation-to $p, q$ and $p x+q y-z$ respectively, say $x^{\prime}, y^{\prime}$ and $z^{\prime}$ for the sake of symmetry in the relations, a new equation in $x^{\prime}, y^{\prime}, z^{\prime}, p^{\prime}, q^{\prime}, r^{\prime}, s^{\prime}, t^{\prime}$ called the reciprocal equation, is obtained, which may be easier of solution than the original equation. Between the equations the following reciprocal relations hold, viz., $x x^{\prime}+y y^{\prime}=z+z^{\prime}, x^{\prime}=p, y^{\prime}=q, x=p^{\prime}, y=q^{\prime}$, and others between the differential co-efficients of the second order, which need not be written down. The equations are thus reciprocal to one another, and from the solution of one that of the other may be obtained by means of the preceding relations.

To these reciprocal relations a geometrical interpretation may be given, which has the advantage of showing very clearly the nature of certain limitations to which reference is about to be made. If $x, y, z$ be regarded as the co-ordinates of a point on a surface $\mathrm{S}$, then $x^{\prime}, y^{\prime}, z^{\prime}$ are the co-ordinates of a point on a surface $S^{\prime}$ which is the polar reciprocal of the surface $S$ with respect to the paraboloid of revolution $x^{2}+y^{2}=2 \approx$, and conversely.

* Philosophical Ma.7azine, March 1892. 
Consider first the process of reciprocation from the purely analytical point of view, without reference to its geometrical significance. In forming the reciprocal equation $p$ and $q$ (written $x^{\prime}$ and $\left.y^{\prime}\right)$ are taken as independent variables, and therefore it is tacitly assumed that $p$ and $q$ are independent. There must therefore be cases in which the method will fail to give, legitimately at least, the full solution of an equation: in some cases, as, for example, that discussed in the paper on waves, already referred to, the most important class of solutions is that in which $p$ is a function of $q$. It is true that when the general solution of an equation can be obtained by this method, those cases in which $p$ and $q$ are not independent may be derived from it by considering them as limiting casesthough it may not always be easy to do so-and the process might be justified in the same way as in other limiting cases ; but it must be remembered that it is by no means the same thing to prove that a theorem holds true up to a limit, and to prove that it holds at the limit.

It is seldom, however, that the general solution of even the reciprocal equation can be obtained in finite terms: generally only a series of particular solutions can be obtained, or, if the reciprocal equation is linear, a general solution made up from these. From such particular solutions no solutions of the original equation in which $p=f(q)$ can be obtained, and from a general solution made up from them such a solution could in general only be derived with great difficulty. Hence, even though a logically rigorous method be not insisted on, the method will generally fail in practice to give this important class of solutions.

The nature of the restriction is made very clear by taking the geometrical point of view. If a surface $S$ is the locus of points whose co-ordinates are so related as to give a solution of the original equation, $S$ may for brevity be said to give a solution of the equation : then its reciprocal $\mathrm{S}^{\prime}$ gives a solution of the reciprocal equation. Now if $p=f(q), \mathrm{S}$ will be a developable surface, and its polar reciprocal $S^{\prime}$ will degenerate into a line, the locus of the poles of its tangent planes; and conversely. But if $\mathrm{S}^{\prime}$ is a line its co-ordinates cannot well be spoken of as satisfying any partial differential equation, for $p^{\prime} q^{\prime} r^{\prime} s^{\prime} t^{\prime}$ are essentially indeterminate. Thus, then, it is clear that solutions of the type $p=f(q)$ can only be got by the method of reciprocation by considering line solutions of the reciprocal equation: such lines can only, of course, be regarded as giving solu- 
tions of an equation when regarded as limiting forms of surfaces which give solutions.

The general conclusion following from these considerations may therefore be thus stated :-The method of solution of partial differential equations by reciprocation can only be regarded as theoretically complete when the deduction of solutions of the type which gives $p=f(q)$ as limiting cases of the general solution is fully justified : but in practice, even though the theoretical completeness of the method of reciprocation be assumed, it should be supplemented by an independent investigation of solutions of the limiting type $p=f(q)$. It should be noticed that Poisson has given a method for obtaining solutions of this type of a certain class of equations: a class to which, it should further be remarked, the method of reciprocation is generally very applicable.

In exemplification of the foregoing discussion, it is desirable to add one or two examples.

Take first the frequently occurring equation

$$
q^{2} v-2 p q^{s}+p^{2} t=0 \ldots \quad \ldots \quad \ldots
$$

The transformed or reciprocal equation is therefore

$$
x^{\prime 2} r^{\prime}+2 x^{\prime} y^{\prime} s^{\prime}+y^{\prime 2} t^{\prime}=0 \quad \ldots \quad \ldots
$$

of which the well known solution is

$$
\begin{array}{rlr} 
& z^{\prime}=x^{\prime} f_{1}\left(y^{\prime} \mid x^{\prime}\right)+f_{2}\left(y^{\prime} \mid x^{\prime}\right) \quad \ldots & \ldots \\
x & =p^{\prime}=\frac{\delta z^{\prime}}{\delta x^{\prime}}=f_{1}-\frac{y^{\prime}}{x^{\prime}} f_{1}^{\prime}-\frac{y^{\prime}}{x^{\prime 2}} f_{2}^{\prime} \ldots \\
& y=q^{\prime}=\frac{\delta z^{\prime}}{\delta y^{\prime}}=f_{1}^{\prime}+\frac{1}{x^{\prime}} f_{2}^{\prime} \quad \ldots \quad \ldots \\
\therefore & z=x x^{\prime}+y y^{\prime}-z^{\prime}=-f_{2}\left(y^{\prime} \mid x^{\prime}\right) \quad \ldots
\end{array}
$$

This gives

But (4) and (5) give

$$
x+y y^{\prime} \mid x^{\prime}=f_{2} \quad \ldots \quad \ldots \quad \ldots
$$

which, by means of $(6)$ gives, changing the arbitrary functions,

$$
x+y \mathrm{~F}_{1}(z)=\mathrm{F}_{2}(z) \quad \ldots \quad \ldots \quad \ldots
$$

which is therefore the general solution of the given equation (1), but may be more symmetrically written

$$
z=x \phi(z)+y \psi(z) \quad \ldots \quad \ldots \quad \ldots
$$

Next seek solutions of the type $p=f(q)$, by Poisson's method. 
Regarding $p$ as a function of $q$ in (1), it may be written

whence

$$
\begin{aligned}
& q^{2} d p^{2}-2 p q d p . d q+p^{2} d q^{2}=0 \quad \ldots \\
& \begin{array}{ccccc} 
& q d p-p d q=0 & \ldots & \ldots & \ldots \\
\therefore \quad \mathrm{A} p & =q \quad \ldots & \ldots & \ldots & \ldots \\
\therefore \quad z & =\mathrm{F}(x+\mathrm{A} y) & \ldots & \ldots & \ldots
\end{array}
\end{aligned}
$$

the solution sought, and which it may be noted is what the general solution (8) above reduces to when $F_{1}(z)$ is taken $=\mathbf{A}$, (or $\psi=\mathbf{A} \phi$ in $(9)$ ).

This example has been taken to show how the special solution may be deduced as a limiting case of the general solution, when that is known; and to show also how easily it may be got by a direct process. It is to be carefully noted that it cannot be derived from anything less than a general solution; it makes $y^{\prime} / x^{\prime}$ in $(7)$ constant, and so is not derivable from any true determinate solution of the reciprocal equation (2).

Consider next an equation whose general solution cannot be obtained in finite terms; for example,

$$
r-p^{2} t=0
$$

Seek first by Poisson's method the solutions in which $q$ and $p$ are not independent : on this hypothesis (14) gives

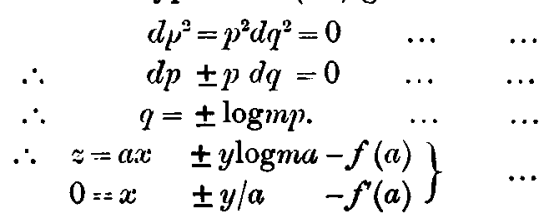

Thus there are two sets of such solutions, got by taking the upper or the lower signs throughout in (18), in which $a$ may be eliminated between the pair of equations, or retained as a variable parameter, as may be most convenient.

These two sets of special solutions have thus been almost immediately obtained by recognising directly the possibility of their existence. Consider next what can be done towards the solution of (14) by the method of reciprocation which may be said to ignore such solutions.

The reciprocal equation is

$$
x^{\prime 2} r^{\prime}-t^{\prime}=0 \quad \ldots \quad \ldots \quad \ldots
$$

This cannot be solved in finite terms, but

$$
z^{\prime}=\mathrm{C}^{\prime m^{\prime \prime} \epsilon^{n y}} \quad \ldots \quad \ldots \quad \ldots
$$


is obviously a particular solution, provided that

$$
m^{2}=n(n-1) \ldots \quad \ldots \quad \ldots
$$

Hence the general solution of $(19)$ is given by

$$
z^{\prime}=\Sigma \mathrm{C}_{m} x^{\prime n} \epsilon^{n y^{\prime}}
$$

in which all values of $m$ and $n$ may be taken, subject to the condition (21). To get the general (or any) solution of (14) from this, the reciprocal relations between the equations have to be used.

Thus

$$
\begin{aligned}
& \boldsymbol{x}=\boldsymbol{p}^{\prime}=\Sigma^{\prime} m \mathrm{C}_{m} x^{\prime m-1} \boldsymbol{\epsilon}^{n y^{\prime}} \quad \ldots \quad \ldots \\
& y=q^{\prime}=\sum n \mathrm{C}_{m} x^{\prime m} \quad \epsilon^{n y} \quad \ldots \quad \ldots \\
& z=x x^{\prime}+y y^{\prime}-z^{\prime}=\Sigma \mathrm{C}_{m}\left(m+n y^{\prime}-1\right) x^{\prime m^{n} \epsilon^{n y^{\prime}}} \quad \ldots
\end{aligned}
$$

These three equations (22), (24), (25), the condition (21) being understood, may be regarded as together forming the general solution of (14), $x^{\prime}$ and $y^{\prime}$ being regarded as variable parameters, which may in special cases be eliminated between these three equations if found desirable.

Now in some limiting form these three equations will presumably reduce so as to give the special solution (18), but it is not easy to see in what particular manner. From (18), in fact, it follows in virtue of the reciprocal relations, that

whence

$$
\begin{aligned}
x^{\prime}=p & =\quad a \\
y^{\prime}=q & = \pm \log m a \\
z^{\prime}=x x^{\prime}+y y^{\prime}-z & =f(a) \\
y^{\prime}= \pm \log m x^{\prime} & =\mathrm{F}\left(z^{\prime}\right)
\end{aligned}
$$

showing that the solutions of (19) which give by reciprocation those of (18), are given by the lines determined by (26). It is evident, however, that (26) can only in a very guarded sense be regarded as giving a solution of (19) at all : it corresponds to none of the particular solutions of the type (20), and it is not easy to see how to get at it as a limiting form of (22).

Thus, finally, we see that it is practically impossible to arrive at any of the important solutions included in (18), either in their general form or as particular cases of it, by the method of reciprocation. In the case considered, regarding (14) as referring to an important problem in the propagation of sound, it may be said that the case missed by reciprocation is physically the most important : it is needless to multiply examples, but further instances may be found in the equations of wave motion which are discussed in the paper alrèady cited. 